\title{
Scheduling optimization of a flexible manufacturing system using a modified NSGA-II algorithm
}

\author{
Nidhiry, N.M. ${ }^{\mathrm{a},{ }^{*}}$, Saravanan, R. ${ }^{\mathrm{b}}$ \\ ${ }^{a}$ Department of Mechanical Engineering, Karapagam University, Coimbatore, Tamil Nadu, India \\ ${ }^{\mathrm{b}}$ Department of Mechanical Engineering, Sri Krishna College of Technology, Coimbatore, Tamil Nadu, India
}

\begin{abstract}
A B S T R A C T
The Flexible Manufacturing System (FMS) belongs to the class of production systems in which the main characteristic is the simultaneous execution of several processes and sharing a finite set of resources. Nowadays, FMS must attend to the demands of market needs for personalized products. Consequently the life-cycle of a product tends to be shorter and a greater variety of products must be produced in a simultaneous manner. The FMS considered in this work has $16 \mathrm{CNC}$ machine tools for processing 80 varieties of products. Since the minimizing of a machine's idle time and thus the minimizing of total penalty costs are contradictory objectives, the problem has a multi-objective nature. The objective of this research was to develop a modified nondominated sorting genetic algorithm (NSGA-II) for multi-objective optimization. The research will then evaluate and discuss the performance of the modified NSGA-II against the original NSGA-II. The existing NSGA II has been modified in order to improve the global optimal front and reduce the computational effort. The result has been compared with the existing NSGA-II, cuckoo search (CS), particle swarm optimization algorithm (PSO), etc. and it was found that the proposed approach was superior.
\end{abstract}

\author{
ARTICLE INFO \\ Keywords: \\ Flexible manufacturing system \\ Scheduling optimization \\ Multi-objective optimization \\ NSGA-II \\ Modified NSGA-II \\ *Corresponding author: \\ nidhishnidhiry@gmail.com \\ (Nidhiry, N.M.) \\ Article history: \\ Received 20 October 2013 \\ First Revision 16 June 2014 \\ Second Revision 16 August 2014 \\ Accepted 19 August 2014
}




\section{References}

[1] Guo, Z.X., Wong, W.K., Leung, S.Y.S., Fan, J.T., Chan, S.F. (2008). A genetic-algorithm-based optimization model for scheduling flexible assembly lines, The International Journal of Advanced Manufacturing Technology, Vol. 36, No. 1-2, 156-168, doi: 10.1007/s00170-006-0818-6.

[2] Tiwari, M.K, Vidyarthi, N.K. (2000). Solving machine loading problems in flexible manufacturing system using a genetic algorithm based heuristic approach, International Journal of Production Research, Vol. 38, No. 14, 33573384, doi: $10.1080 / 002075400418298$.

[3] Kumar, A.V.S.S., Veeranna, V., Prasad, B.D., Sarma, B.D. (2010). Optimization of FMS scheduling using nontraditional techniques, International Journal of Engineering Science and Technology, Vol. 2, No. 12, 7289-7296.

[4] Kumar, R., Tiwari, M.K., Shankar, R. (2003). Scheduling of flexible manufacturing systems: an ant colony optimization approach, In: Proceedings of the Institution of Mechanical Engineers, Part B: Journal of Engineering Manufacture, Vol. 217, No. 10, 1443-1453, doi: 10.1243/095440503322617216.

[5] Toker, A., Kondakci, S., Erkíp, N. (1994). Job shop scheduling under a non-renewable resource constraint, The Journal of the Operational Research Society, Vol. 45, No. 8, 942-947, doi: 10.2307/2584018.

[6] Hoitomt, D.J., Luh, P.B., Pattipati, K.R. (1993). A practical approach to job-shop scheduling problems, Robotics and Automation: IEEE Transactions on, Vol. 9, No. 1, 1-13, doi: 10.1109/70.210791.

[7] He, W., Kusiak, A. (1992). Scheduling manufacturing systems, Computers in Industry, Vol. 20, No. 2, 163-175, doi: 10.1016/0166-3615(92)90050-W.

[8] Lee, D.Y., DiCesare, F. (1994). Scheduling flexible manufacturing systems using Petri nets and heuristic search, Robotics and Automation: IEEE Transactions on, Vol. 10, No. 2, 123-132, doi: 10.1109/70.282537.

[9] Shnits, B., Sinreich, D. (2006). Controlling flexible manufacturing systems based on a dynamic selection of the appropriate operational criteria and scheduling policy, International Journal of Flexible Manufacturing Systems, Vol. 18, No. 1, 1-27, doi: 10.1007/s10696-006-9001-5.

[10] Yu, M.-C., Greene, T.J. (2006). A simulation analysis of controlling rules for flexible pull systems, International Journal of Manufacturing Research, Vol. 1, No. 3, 314-331, doi: 10.1504/IJMR.2006.011961.

[11] Jerald, J., Asokan, P., Prabaharan, G., Saravanan, R. (2004). Scheduling optimisation of flexible manufacturing systems using particle swarm optimisation algorithm, The International Journal of Advanced Manufacturing Technology, Vol. 25, No. 9-10, 964-971, doi: 10.1007/s00170-003-1933-2.

[12] Saravana Sankar, S., Ponnanbalam, S.G., Rajendran, C. (2003). A multiobjective genetic algorithm for scheduling a flexible manufacturing system, The International Journal of Advanced Manufacturing Technology, Vol. 22, No. 3-4, 229-236, doi: $10.1007 / \mathrm{s} 00170-002-1464-2$.

[13] Burnwal, S., Deb, S. (2013). Scheduling optimization of flexible manufacturing system using cuckoo search-based approach, The International Journal of Advanced Manufacturing Technology, Vol. 64, No. 5-8, 951-959, doi: 10. 1007/s00170-012-4061-z.

[14] Udhayakumar, P., Kumanan, S. (2010). Sequencing and scheduling of job and tool in a flexible manufacturing system using ant colony optimization algorithm, The International Journal of Advanced Manufacturing Technology, Vol. 50, No. 9-12, 1075-1084, doi: 10.1007/s00170-010-2583-9.

[15] Kumar, M.V.S, Janardhana, R., Rao, C.S.P. (2011). Simultaneous scheduling of machines and vehicles in an FMS environment with alternative routing, The International Journal of Advanced Manufacturing Technology, Vol. 53, No. 1-4, 339-351, doi: 10.1007/s00170-010-2820-2.

[16] Saravanan, M., Haq, A.N. (2008). Evaluation of scatter-search approach for scheduling optimization of flexible manufacturing systems, The International Journal of Advanced Manufacturing Technology, Vol. 38, No. 9-10, 978986, doi: 10.1007/s00170-007-1134-5.

[17] Nakamoto, F.Y., Miyagi, P.E., Santos Filho, D.J. (2008). Resources allocation control in flexible manufacturing systems using the deadlock avoidance method, In: Proceedings of ABCM Symposium Series in Mechatronics, Vol. 3, 454-460.

[18] Jian, C.F., Wang, Y. (2014). Batch task scheduling-oriented optimization modelling and simulation in cloud manufacturing, International Journal of Simulation Modelling, Vol. 13, No. 1, 93-101, doi: 10.2507/IJSIMM13(1)CO2.

[19] Reddy, B.S.P., Rao, C.S.P (2011). Flexible manufacturing systems modelling and performance evaluation using AutoMod, International Journal of Simulation Modelling, Vol. 10, No. 2, 78-90, doi: 10.2507/IJSIMM10(2)3.176.

[20] Deb, K., Pratap, A., Agarwal, S., Meyarivan, T. (2002). A fast and elitist multiobjective genetic algorithm: NSGA-II, IEEE Transactions on Evolutionary Computation, Vol. 6, No. 2, 182-197, doi: 10.1109/4235.996017.

[21] Deb, K. (2001). Multi-objective optimization using evolutionary algorithms, Wiley, New York, USA.

[22] Nidhiry, N.M., Saravanan, R. (2014). Scheduling optimization of FMS using NSGA-II, Advances in Industrial Engineering and Management, Vol. 3, No. 1, 63-72, doi: 10.7508/AIEM-V3-N1-63-72.

[23] Nidhiry, N.M., Saravanan, R. (2014). FMS scheduling optimization using modified NSGA-II, International Journal of Mechanical and Production Engineering, Vol. 2, No. 2, 1-6. 


\title{
Optimizacija terminiranja opravil v prilagodljivem obdelovalnem sistemu $z$ uporabo modificiranega algoritma NSGA-II
}

\author{
Nidhiry, N.M..$^{\mathrm{a},{ }^{*}}$, Saravanan, R. ${ }^{\mathrm{b}}$ \\ a Department of Mechanical Engineering, Karapagam University, Coimbatore, Tamil Nadu, India \\ ${ }^{b}$ Department of Mechanical Engineering, Sri Krishna College of Technology, Coimbatore, Tamil Nadu, India
}

\section{POVZETEK}

Glavna značilnost prilagodljivega obdelovalnega sistema (angl. Flexible Manufacturing System - FMS) je hkratno izvajanje številnih izdelovalnih postopkov in skupna raba omejenega števila proizvodnih virov. FMS mora slediti zahtevam trga, ki so usmerjene $\mathrm{k}$ individualizaciji izdelkov. Zaradi tega se skrajšuje življenjski cikel izdelka, poleg tega pa se hkratno izdeluje v vedno več različicah. V delu obravnavamo FMS, ki ga sestavlja $16 \mathrm{CNC}$-strojev, ki so namenjeni za izdelavo 80 različic izdelkov. Ker sta minimizacija časa, ko stroj ne dela in zatorej minimizacija celotnih stroškov nasprotujoči si zahtevi, ima problem večkriterijsko naravo. Cilj pričujočega dela je bil razviti modificiran genetski algoritem, temelječ na nedominiranem razvrščanju (NSGA-II), s katerim smo rešili omenjen problem večkriterijske optimizacije. Modifikacije izvirnega algoritma se nanašajo predvsem na izboljšanje globalne optimalne fronte in skrajšanje potrebnega časa obdelave. $V$ raziskavi je narejena tudi primerjava zmogljivosti modificiranega algoritma NSGA-II z izvirno metodo NSGA-II, poleg tega pa še z metodami kukavičje iskanje (angl. Cuckoo Search - CS), optimizacijo z rojem delcev (angl. Particle Swarm Optimization - PSO) itd. Ugotovili smo, da predlagan pristop daje boljše rezultate od preostalih metod.
\end{abstract}

\section{PODATKI O ČLANKU}

Ključne besede:

Prilagodljivi obdelovalni sistem

Optimizacija terminiranja

Večkriterijska optimizacija

Algoritem NSGA-II

Modificiran algoritem NSGA-II

* Kontaktna oseba:

nidhishnidhiry@gmail.com

(Nidhiry, N.M.)

Zgodovina članka:

Prejet 20. oktobra 2013

Prvič popravljen 16. junija 2014

Drugič popravljen 16. avgusta 2014

Sprejet 19. avgusta 2014

(C) 2014 PEI, University of Maribor. All rights reserved. 